\title{
Espiritualidad y cuidado humanizado de los estudiantes de Enfermería de una universidad privada, Perú, 2020
}

\author{
Spirituality and humanized care of nursing students from a private university, Peru, \\ 2020
}

\author{
Glendy Larico Calla ${ }^{1}$, Dennis Mamani Quispe ${ }^{2}$
}

\begin{abstract}
RESUMEN
Objetivo: Determinar la relación entre la espiritualidad y el cuidado humanizado que brindan los estudiantes de Enfermería de una universidad privada de Perú. Metodología: De enfoque cuantitativo, diseño no experimental, tipo correlacional y de corte transversal. La muestra fue obtenida por muestreo probabilístico llegando a ser conformado por 117 estudiantes de enfermería. Los instrumentos utilizados fueron: la escala de autoeficacia del cuidado creado por Coates (1992), adaptada al español por Poblete, Valenzuela, \& Merino, (2012) con un coeficiente de alpha de Cronbach de 0.87 y el cuestionario de espiritualidad creado por Parsian y Dunning, (2009), adaptado y traducido al español por Díaz, Muñoz Sánchez y Vargas (2012) con un coeficiente de alpha de Cronbach de 0.88. Resultados: Existe relación entre las variables espiritualidad y cuidado humanizado con un $\mathrm{p}$ valor de 0.004, según el estadístico de correlación Spearman. Del mismo modo, se encontró relación entre las dimensiones de la espiritualidad (autoconciencia, importancia de las creencias espirituales, prácticas espirituales y necesidades espirituales) con el cuidado humanizado. Con respecto a los datos descriptivos el $84,6 \%$ de los estudiantes tiene un nivel de espiritualidad alto y el $54,7 \%$ tiene un nivel de cuidado humanizado alto. Conclusión: Existe relación entre las variables de estudio.
\end{abstract}

Palabras clave: Espiritualidad, cuidado humanizado, estudiantes de enfermería.

\begin{abstract}
Objective: To determine the relationship between spirituality and humanized care provided by nursing students from a private university in Peru. Methodology: Quantitative approach, non-experimental design, correlational type and cross section. The sample was obtained by probabilistic sampling, reaching the composition of 117 nursing students. The instruments used were: Caring Efficacy Scale created by Coates (1992), adapted to Spanish by Poblete, Valenzuela, \& Merino, (2012) with a Cronbach's alpha coefficient of 0.87 and the spirituality questionnaire created by Parsian and Dunning, (2009), adapted and translated into Spanish by Díaz, Muñoz Sánchez and Vargas (2012) with a Cronbach alpha coefficient of 0.88 . Results: There is a relationship between the variables spirituality and humanized care with a p-value of 0.004 , according to the Spearman correlation statistic. Similarly, a relationship was founded between the dimensions of spirituality (self-awareness, importance of spiritual beliefs, spiritual practices, and spiritual needs) with humanized care. Regarding the descriptive data, $84.6 \%$ of the students have a high level of spirituality and $54.7 \%$ have a high level of humanized care. Conclusion: There is a relationship between the study variables.
\end{abstract}

Keywords: Spirituality, humanized care, nursing students.

${ }^{1}$ Universidad Peruana Unión, Lima, Perú.

Orcid ID: 0000-0002-5368-8369

${ }^{2}$ Clínica Good Hope, Lima, Perú.

Orcid ID: 0000-0002-8248-5309 


\section{INTRODUCCIÓN}

El significado y esencia del cuidado humanizado vienen de la raíz de la palabra "humanidad" definida como sensibilidad, compasión, bondad hacia los semejantes (RAE, 2020).

Rodríguez y Honores (2016) definen el cuidado humanizado como la expresión constante, incondicional y atenta frente al paciente; es contribuir a la recuperación de su autonomía e independencia; es la preocupación y el esfuerzo para alcanzar el máximo bienestar físico, mental y espiritual. Para alcanzar este bienestar son necesarias la comprensión, la aceptación, la tolerancia, la comunicación terapéutica y la escucha activa.

En los países latinoamericanos, la encargada de gestionar este cuidado es la enfermera, ya que Enfermería tiene como eje principal de su quehacer el cuidado en la atención y prestación de servicios en salud, con un compromiso no observado en otra disciplina o profesión. Sin embargo, en estos últimos años, donde se ha evidenciado el desarrollo de tecnológico más grande en el área de salud, surge el fenómeno de deshumanización, donde la persona pasa a ser simplemente objeto de la aplicación de la medicina y el enfermo pasa a ser la enfermedad. Este fenómeno hace que el trabajo de enfermería se realice de forma mecanizada, tornando su trabajo invisible en los campos clínicos y generando así insatisfacción laboral (Monje et al., 2018).

Albornoz y Chiquez (2018) afirman que en Perú, en los hospitales nacionales, seguro social y las diversas clínicas, el profesional de Enfermería continua dando prioridad al aspecto administrativo, documental y seguimiento de protocolos establecidos, dejando de lado el lado humano y la sensibilidad que debe tener el profesional, esta afirmación es respalda por varias investigaciones en diversas ciudades del Perú, dentro de ellas (Calsin, 2017) en Puno demostró que los pacientes consideran deficiente el cuidado humanizado por parte de las enfermeras, por otro lado, (Paccotaipe, 2018) afirma que en los hospitales de Lima norte los pacientes consideran regular el cuidado recibido por parte del profesional de enfermería, demostrando así que no están satisfechos con el cuido recibido.

Posibles causantes de la deshumanización son la sobrecarga laboral, el ambiente, las características personales; como la autoestima, valores, espiritualidad, principios y entre otros (Albornoz y Chiquez 2018).

Adicionalmente, Melgar y Estuco (2014) sustentan que el cuidado holístico o también llamado humanizado solamente es posible cuando el enfermero está consciente de sí mismo y cuando su ser está integrado y armonioso, para poder cuidar de un ser humano en las dimensiones biológica, emocional, espiritual; el profesional de Enfermería necesita cuidar de sí mismo a través de la continua búsqueda de lo trascendental, armonía e integridad. Los autores sustentan que cuanto mayor sea la espiritualidad del profesional de enfermería, mejor será la percepción del cuidado humanizado por parte del paciente.

Por su parte, Silva (2015) infiere que, al hablar de espiritualidad, los estudiantes de enfermería lo relacionan con el cuidado humanizado. Resultados similares fueron encontrados por (Evangelista et al., 2016) quien al entrevistar a profesionales de enfermería concluyó que la espiritualidad del enfermero se torna una herramienta para el cuidado que brinda.

Para Bennett y Thompson (2015) la mejor forma de enseñar a brindar un cuidado holístico a los estudiantes de enfermería, es promover la propia espiritualidad de los mismos. Esta teoría fue probada por Ross et al. (2018) quien demostró que se puede enseñar a los alumnos espiritualidad, y así incrementar su cuidado humanizado; ya que el mejor momento para pulir e reforzar estos aspectos de forma oportuna, es en el pregrado, para que cuando ingrese al mundo laboral brinde un cuidado humanizado de manera natural espontánea.

Sin embargo, Babamohamadi, Ahmadpanah y Ghorbani (2018) en Irán evaluaron la espiritualidad y cuidado holístico entre las enfermeras y estudiantes de enfermería, pero solamente el $33.9 \%$ obtuvo un nivel alto. En cambio, Daghan (2017) en Turquía demostró que los estudiantes de enfermería en su mayoría tienen un nivel medio en espiritualidad y cuidado holístico. Adicionalmente, Alva y Castillo (2018) hallaron en la misma casa de estudio, de esta investigación, que la mayoría de estudiantes de Medicina poseen un nivel de espiritualidad bajo.

Es por ello que se ha considerado trascendental estudiar esta problemática con el objetivo de 
analizar y brindar información sobre la relación de estas variables.

\section{METODOLOGÍA}

Esta investigación es de enfoque cuantitativo, de diseño no experimental, de corte transversal, y de tipo correlacional.

\section{Participantes}

La población de estudio estuvo conformada por los 168 estudiantes del 3 al 5 año de la escuela profesional de enfermería de la sede y filial de una universidad privada de Perú. La selección de la muestra se obtuvo mediante fue probabilístico aleatorio simple. La muestra, estuvo conformada por los 117 estudiantes.

\section{Instrumentos}

\section{Cuestionario de Espiritualidad}

Fue creado por Parsian y Dunning, (2009) en Australia, con el objetivo medir el constructo espiritualidad, está constituido por 4 dimensiones: autoconciencia, importancia de las creencias espirituales, prácticas espirituales y necesidades espirituales. En su versión original, obtuvo una confiabilidad alfa de Cronbach de 0.94. Posteriormente, fue adaptado y traducido al español en Colombia por Díaz, Muñoz Sánchez y Vargas (2012) donde obtuvo una confiabilidad alfa de Cronbach de 0.88. En Perú, fue validado por Tacilla y Robles, (2015) donde obtuvieron una validez a través de $\mathrm{V}$ de Aiken $=0.75$ y una confiabilidad de alpha de Cronbach $=0.952$ lo cual indica una alta consistencia, consta de 29 preguntas en escala de Likert con puntuaciones desde 1 al 4.

\section{Caring Efficacy Scale (CES)}

Para medir la variable de cuidado humanizado fue utilizado el CES, que en español significa escala de autoeficacia del cuidado, fue creado por Carolle Coates (1992) en la Universidad de Colorado, U.S.A. Tiene por objetivo medir la percepción de autoeficacia que tiene la enfermera al otorgar sus cuidados y relacionarse con sus pacientes; esta escala está basada en la Teoría Autoeficacia de Barunda y en la Teoría Transpersonal del cuidado humano de Jean Watson, incorporando los 10 factores de cuidado de la teoría, posteriormente, adaptada al español por Poblete, Valenzuela \& Merino (2012), en Perú, fue adaptado por Casimiro y Palma (2016) en su investigación titulada "Calidad de cuidados humanizados que brinda el profesional de enfermería en los servicios de hospitalización de un hospital público de Huánuco-2016", donde obtuvo un coeficiente de Cronbach de 0.87 .

EI CES está compuesto por 30 ítems, balanceados entre 15 negativos y 15 positivos, de formato Likert con puntuaciones desde -3 a +3 .

\section{Procesamiento de datos}

Para la recolección de datos del estudio, se procedió a solicitar la autorización a la institución a través de una carta dirigida al comité de ética e investigación de la sede y filial. Una vez obtenida la autorización, se procedió a encuestar a los alumnos vía online y física en las instalaciones de la universidad, entre los meses de marzo mayo del 2020.

El procesamiento de datos comenzó con el ingreso, codificación y limpieza de datos en el estadístico IBM SPSS 22. Para el análisis de los datos descriptivos se realizaron tablas de frecuencia y para el análisis inferencial, primeramente, para determinar la normalidad se realizó la prueba de Kolmogorov-Smirnov, donde se obtuvo un p-valor menor a 0.05 , por lo que se utilizó la prueba de correlación de rho de Spearman, prueba no paramétrica, debido a la naturaleza de las variables.

\section{RESULTADOS}

En la tabla 1 podemos observar la relación entre las variables de estudio, el estadístico coeficiente de correlación Spearman evidencia correlación de rho=.266 y un $\mathrm{p}$ - valor de 0.004 , en el cual muestra una significancia menor de 0.05 , lo que permite rechazar la Ho, quedando demostrado que existe relación entre el nivel de espiritualidad frente al cuidado humanizado en estudiantes de Enfermería de una universidad privada de Perú. 
Tabla 1

Relación entre la espiritualidad y el cuidado humanizado que brindan los estudiantes de Enfermería de una universidad privada, Perú, 2020

\begin{tabular}{|c|c|c|c|c|c|}
\hline \multirow{2}{*}{ Espiritualidad } & & \multicolumn{3}{|c|}{ Cuidado humanizado } & \multirow{2}{*}{ Total } \\
\hline & & Deficiente & Regular & Alto & \\
\hline \multirow[t]{2}{*}{ Bajo } & $\mathrm{n}$ & 3 & 8 & 7 & 18 \\
\hline & $\%$ & $2,6 \%$ & $6,8 \%$ & $6,0 \%$ & $15,4 \%$ \\
\hline \multirow[t]{2}{*}{ Alto } & $\mathrm{n}$ & 14 & 28 & 57 & 99 \\
\hline & $\%$ & $12,0 \%$ & $23,9 \%$ & $48,7 \%$ & $84,6 \%$ \\
\hline \multirow[t]{2}{*}{ Total } & $\mathrm{n}$ & 17 & 36 & 64 & 117 \\
\hline & $\%$ & $14,5 \%$ & $30,8 \%$ & $54,7 \%$ & $100,0 \%$ \\
\hline
\end{tabular}

rho=.266; $p=.004$

En la tabla 2, se observa que del $100 \%$ de encuestados, $84,6 \%$ tiene un nivel de espiritualidad alto y $15,4 \%$ bajo.

Tabla 2

Nivel de espiritualidad de los estudiantes de Enfermería de una universidad privada, Perú, 2020

\begin{tabular}{lcc} 
Variable & N & $\%$ \\
Espiritualidad & 18 & 15,4 \\
Bajo & 99 & 84,6 \\
Alto & & \\
Autoconciencia & 19 & 16,2 \\
Bajo & 98 & 83,8 \\
Alto & & \\
Importancia de las creencias espirituales & 29 & 24,8 \\
Bajo & 88 & 75,2 \\
Alto & & \\
Practicas espirituales & 19 & 16,2 \\
Bajo & 98 & 83,8 \\
Alto & & 20,5 \\
Necesidades espirituales & 24 & 79,5 \\
Bajo & 93 & 100,0 \\
Alto & 117 & \\
Total & & \\
\hline
\end{tabular}

En la tabla 3 , se observa que del $100 \%$ de estudiantes encuestados $54,7 \%$ tiene un nivel de cuidado humanizado alto; por otro lado, 30,8\% regular y un $14 \%$ deficiente. 
Tabla 3

Nivel de cuidado humanizado que brindan los estudiantes de Enfermería de una universidad privada, Perú, 2020

\begin{tabular}{lll} 
Cuidado humanizado & N & $\%$ \\
Deficiente & 17 & 14,5 \\
Regular & 36 & 30,8 \\
Alto & 64 & 54,7 \\
Total & 117 & 100,0 \\
\hline
\end{tabular}

\section{DISCUSIÓN}

Para la Organización mundial de la salud (2020) el ser humano está compuesto por una parte biológica, mental y social. Sin embargo, actualmente, está demostrado que el ser humano es un ser espiritual, a pesar que por mucho tiempo la cultura occidental ha intentado aislar el cuerpo del alma, como si fueran elementos en oposición, ya que a través de la espiritualidad construimos nuestra propia cosmovisión, sabemos quiénes somos, de dónde venimos y a dónde vamos. Obteniendo respuestas a las preguntas existenciales, logramos encontrar armonía y sentimiento de pertenencia con nosotros mismos, con el otro, con la naturaleza y con la vida y con lo trascendental, llámese, Dios, Buda, o cualquier otro nombre (Gallegos y Herrera, 2008) y (Koenig, McCullough y Larson, 2001).

Poseer una buena espiritualidad brinda muchos beneficios al ser humano, estos fueron demostrados a través de muchos estudios; dentro de estos, tenemos a Salgado (2014) quien, tras una revisión minuciosa de los beneficios de la espiritualidad, afirma que la espiritualidad contribuye a una mejor autoestima, fortaleza y esperanza, relacionado a mayor satisfacción con la vida y la capacidad para perdonar. Además, es un apoyo emocional y social, que promueve valores pros sociales, asociados a menor consumo de narcóticos y menor tendencia a fumar. Por consiguiente, ayuda a una mejor salud física y psicológica, como ayuda de prevención y mejoramiento de enfermedades; también, disminuye la depresión, ansiedad y el estrés, ayudando al enfrentamiento de enfermedades y el temor a la muerte.

Es por esta razón que el profesional de enfermería necesita cuidar y fortalecer este aspecto de su ser, ya que parte esencial de su labor es brindar cuidado humanizado a los pacientes o usuarios. Al referirnos al cuidado humanizado, se relacionan con el saber científico, la pericia técnica y la relación existente entre enfermeropaciente, donde ambos se aceptan en el proceso de interacción, de forma afectiva y respetuosa sin dejar de lado la autonomía. Es un proceso recíproco como resultado durante el momento del cuidado de forma holística (Guerrero et al., 2015). Por consiguiente, la falta de compromiso, dedicación, respeto, empatía y cariño con que brindan su cuidado, reflejan que se adolece de un cuidado humanizado (Álvarez y Román, 2015).

Al analizar la relación entre las variables propuestas aceptamos la hipótesis alternativa general que establece que existe relación entre la espiritualidad y el cuidado humanizado, obteniendo un p-valor de 0.004 a través del coeficiente de correlación Spearman.

Estos resultados guardan relación con Melgar y Estuco (2014) quien es su investigación reveló que existe relación entre la espiritualidad de las enfermeras y la percepción del cuidado humanizado por parte de sus pacientes, con $\mathrm{p}$ valor de $P=0.039$. En conclusión, cuanto mayor sea la espiritualidad de la enfermera, mayor será el nivel de percepción de los pacientes.

Adicionalmente, Silva (2015), al realizar su investigación de enfoque cualitativo, concluye que al hablar de espiritualidad los estudiantes de enfermería la relacionan con un cuidado humanizado.

Tal como postula, Afolabi (2017) esta relación se debe a que la espiritualidad favorece la sensibilidad, empatía y la habilidad intrapersonal e interpersonal, características fundamentales del cuidado humanizado. Este tipo de comportamiento basado en el amor, es lo que Paredes(2017)consideracomovalores básicos de Enfermería, los cuales son: amor, comunicación, confiabilidad, honestidad, integridad, lealtad, liderazgo, optimismo, paciencia, responsabilidad, 
solidaridad, tolerancia, veracidad, respeto. Son los mismos valores mencionados en la Biblia, en Gálatas 5: 22-23, como los frutos de espíritu. Estos son valores que nacen en la persona que tiene al espíritu santo en ella, es decir, una espiritualidad alta.

El resultado descriptivo, en cuanto a la espiritualidad de los estudiantes de enfermería, muestra que del $100 \%$ de encuestaos, $84,6 \%$ tiene un nivel de espiritualidad alto y $15,4 \%$ bajo, siendo la dimensión autoconciencia y prácticas religiosas las más sobresalientes.

Por el contrario, Alva y Castillo (2018), al investigar el nivel de espiritualidad en estudiantes de medicina, de la misma casa de estudios, descubrieron que el $75 \%$ de alumnos tuvo un nivel de espiritualidad bajo y solamente un $25 \%$ alto.

Adicionalmente, Lujan y Moisés (2014) realizaron una investigación que midió el bienestar espiritual en profesionales de Enfermería asistencial en un hospital de Ayacucho. Este demostró que el $76 \%$ de los profesionales de enfermería presentaba un nivel medio de bienestar espiritual y el $24 \%$ presentó un nivel bajo. Resultados similares presentó Daghan (2017), quien realizó un estudio en Turquía, con el fin de saber cómo los estudiantes de enfermería perciben la espiritualidad y cuidado espiritual. El resultado mostró que tenían una percepción espiritual media 3.60 (IQR = 3.55-3.82), lo que demostraba una confusión conceptual.

De la misma manera, Babamohamadi et al. (2018) realizaron un estudio en Irán cuyo objetivo fue medir las actitudes hacia la espiritualidad $y$ el cuidado espiritual entre enfermeras $y$ estudiantes de enfermería iraníes. Los resultados encontrados revelan que solamente el $33.9 \%$ presentó un puntaje alto o deseable, siendo que el $64.3 \%$ obtuvo un puntaje promedio.

Por su parte, Evangelista et al. (2016), en su investigación de enfoque cualitativo, demostró que el profesional de enfermería no tiene una preparación completa en cuanto a la espiritualidad, pudiendo ser esta la causa por la que el profesional de enfermería no demuestra niveles altos de espiritualidad. Apoyando esta teoría, Ross et al. (2018) realizaron un estudio en 8 países de Europa, cuyo objetivo fue medir las percepciones de los estudiantes de enfermería y obstetricia sobre la espiritualidad, el cuidado espiritual y la competencia del cuidado espiritual. Los resultados mostraron la competencia percibida incrementó a lo largo del estudio; este incremento es atribuido a las experiencias de vida, a la enseñanza / discusión en la universidad sobre los temas estudiado, demostrando así de que la competencia de cuidado espiritual; que es parte del cuidado humanizado, se desarrolla en estudiantes de pregrado de enfermería y obstetricia y que las percepciones de espiritualidad y espiritualidad personal de los estudiantes contribuyen a ese desarrollo.

Por su parte, Bennett y Thompson (2015) postulan que en el marco de un cuidado holístico que incluya la espiritual, debe haber un mayor énfasis en la educación que promueva la propia espiritualidad de la enfermera, pues el mayor predictor de la aptitud de los estudiantes para brindar atención espiritual es la percepción de su propia espiritualidad personal. Los estudiantes primero deben evaluar su propia espiritualidad antes de poder relacionarse de alguna manera con la necesidad espiritual de otra persona. He aquí la importancia de conocer el estado espiritual de los estudiantes de enfermería, crear un espacio en donde los estudiantes puedan fortalecer y expresar su espiritualidad y ver cómo esta influye en su vida y el cuidado que brindan. Seguramente, esta es la razón por la que encontramos un alto nivel de espiritualidad en los estudiantes de enfermería en estudio, ya que la institución en donde realizan sus estudios de pregrado brinda un ambiente y diseño curricular propicio para que sus alumnos forjen un nivel alto de espiritualidad.

Otros datos descriptivos encontrados en el estudio revelan que, en la autopercepción del cuidado humanizado de los estudiantes de enfermería, del $100 \%$ de estudiantes encuestados $54,7 \%$ tiene un nivel de cuidado humanizado alto, por otro lado $30,8 \%$ regular y un $14 \%$ deficiente.

Estos resultados guardan relación con los encontrados en el estudio de Romero, Contreras y Moncada (2016) en Cartagena - Colombia, quienes demostraron que el $55,4 \%$ de los pacientes hospitalizados percibieron un nivel excelente en el cuidado humanizado, el $35 \%$ bueno, $7.9 \%$ aceptable y $1.7 \%$ malo.

Casimiro y Palma (2016) realizaron una investigación en el servicio de emergencia en 
un hospital de Huánuco, donde encontró que un $48.6 \%$ de enfermeros percibían como bueno o favorable los cuidados que brindaban, seguido de una calidad de cuidados deficiente con un $36.1 \%$ y un $15.3 \%$ percibían como regular sus cuidados humanizados.

Así también, Ugarte (2017), en Lima, mostró que el $61 \%$ de los pacientes expresan que las enfermeras brindan un buen cuidado humanizado, $36 \%$ regular y $2 \%$ refieren que nunca recibieron un cuidado humanizado.

En contraposición, Calsin (2017) realizó, en Puno, una investigación en el servicio de Gineco - Obstetricia en un hospital Nacional donde indicaban que el $96 \%$ de los pacientes perciben como deficiente el cuidado humanizado que brinda el profesional de enfermería y el $4 \%$ como regular.

Por su parte, Cabana y Delgado (2016), en su investigación en Arequipa, reveló que el $79.5 \%$ de los profesionales de enfermería auto percibía que brindaba un cuidado humanizado considerado como media, $20.5 \%$ como malo y ningún miembro de la población en estudio llegó a tener una autopercepción alta.

Resultados contrarios, también fueron hallados por Moreno et al., (2018) en España, quienes mostraron que el $83,7 \%$ de enfermeros auto percibía que se brindaba un cuidado humanizado bueno y $16.3 \%$ un cuidado humanizado malo.

En cuanto a estudios aplicados en estudiantes de enfermería, tal como se realiza en esta investigación, no se encontraron muchas investigaciones. Sobre este tema, Santos et al. (2018) refieren que existe una escasez de publicaciones y esto refleja la necesidad de invertir en nuevas investigaciones sobre el tema dentro del campo de la Enfermería. Solo, de esta manera, mejoraremos la capacitación $y$, en consecuencia, la práctica y el arte de la Enfermería.

Como menciona Landman (2016), son muchos los factores que fluyen el cuidado humanizado que brinda el enfermero y el cuidado humanizado que percibe el paciente, ya que este cuidado está asociado a experiencias personales, conocimientos científicos, habilidades técnicas, valores y motivación para trabajar por y para otros.
Como argumentan los investigadores mencionados, uno de los aspectos para brindar un cuidado humanizado es la formación científica; esta se desarrolla dentro de aulas. Es necesario que en la formación del futuro profesional de enfermería se le enseñe todo lo necesario para bridar este cuidado. Apoyando esta teoría Gualdrón et al. (2019) afirma que el cuidado humanizado es el resultado de la interrelación disciplinar entre conocimiento y acción. Por esta razón, se considera importante evaluar las condiciones en que se ofrece el cuidado, las actitudes del personal de estudiante y profesional de enfermería frente a los pacientes y familia, como base para el análisis y toma de decisiones.

Aunque de forma progresiva, se ha estado considerando la autopercepción del cuidado del enfermero y siguen siendo predominante la cantidad de estudios que únicamente ve la perspectiva del paciente. Para autores como Bandura (1987), es imprescindible ver la autopercepción de los alumnos y profesionales de enfermería puesto que la autoeficacia es más que la confianza que poseemos en nosotros mismos. Un mecanismo predictivo de la conducta futura influye sobre la conducta, en otras palabras, la autoeficacia hace de la persona sea la productora de su propio comportamiento.

Como vemos en este estudio, casi la mitad de los alumnos auto percibía que brindaba un cuidado humanizado de nivel alto, aunque pocos fueron los que auto percibían un nivel bajo. Es necesario continuar educando y evaluando este aspecto, para así garantizar una correcta formación integral.

En el trabajo de investigación, también se encontró que existe relación entre la dimensión autoconciencia y el cuidado humanizado que brindan los estudiantes de Enfermería con un un $\mathrm{p}$ - valor de 0.004 .

Resultados similares fueron encontrados por Park y Chung (2015), quienes en su investigación encontraron que existe relación entre habilidad de comunicación, la autoestima y la competencia clínica. Concluyeron que, para mejorar la competencia clínica de los estudiantes de enfermería, es necesario mejorar la habilidad de comunicación y la autoestima. De igual manera, Losa et al. (2017), muestra que existe una relación entre una autoestima, el autoconcepto de las enfermeras y sus habilidades sociales. Esta relación puede llegar a afectar de manera 
positiva o negativa la comunicación asertiva con los pacientes.

Frente a estos datos, Pico (2020) afirma que el primer paso para comprender a los demás es tomar autoconciencia y autoconocimiento personal. El autoconocimiento o autoconciencia no suele asociarse con la profesión de enfermería; sin embargo, la forma en que las enfermeras piensan y sienten acerca de sí mismas tiene implicaciones importantes a nivel personal y profesional; en otras palabras, afecta el estado de bienestar propio, la calidad de la atención brindada al paciente, la satisfacción laboral. (Van Eckert et al., 2012)

La espiritualidad brinda al ser humano la oportunidad de conocerse a sí mismo, ya que responde las preguntas ontológicas, además de brindar paz y armonía interna. Adicional a esto, Salgado (2014) demostró que la espiritualidad contribuye a una mejor autoestima e inteligencia emocional.

Por otro lado, se encontró relación entre la dimensión importancia de las creencias espirituales y el cuidado humanizado con un pvalor de 0.016 .

Resultados similares obtuvo Miguel y Vilchez, (2018), quienes demostraron que existe correlación entre la espiritualidad e inteligencia emocional en estudiantes universitarios. Aparte de ello, Arikhman, Machmud, Hasan y Bachtiar (2016) refieren que los trabajadores de centros de salud asocian las habilidades espirituales como un aspecto que conforma las habilidades blandas, las cuales son necesarias para brindar un cuidado holístico.

En el estudio, también se encontró relación entre la dimensión prácticas espirituales y el cuidado humanizado con un p- valor de 0.010. Resultados similares, fueron encontrados por Anchorena (2014), quien mostró que los estudiantes católicos practicantes entre 14 y 17 años presentan mayores niveles de inteligencia emocional-social que los no practicantes.

Según Gonzales (2004), existen dos tipos de prácticas espirituales: las de carácter extrínseco, como la asistencia a denominaciones religiosas; y las de carácter intrínseco, estas son las actividades que permiten al ser humano tener una relación personal con Dios, tales como la fe, oración, lectura personal de las escrituras, entre otras actividades personales.

Por otro lado, White (2012) menciona que las personas que atienden a los enfermos necesitan de oración y estudio de la Palabra, para que su vida sea ejemplo de la Luz de Dios. Al tener una espiritualidad verdadera tendrán la clave para amar a los seres humanos como Dios los ama; no curando solo la enfermedad sino también el alma. Apoyando esta teoría, Quintero (2001) refiere que el cristianismo demuestra su amor a Dios por medio del cuidado y el amor a los pobres y enfermos. Afirma también que la enfermería recibió gran influencia del cristianismo; principalmente valores como la solidaridad, el respeto a la vida y el amor al prójimo.

Adicionalmente, se encontró relación entre la dimensión necesidades espirituales y el cuidado humanizado con un p- valor de 0.012,

Al respecto, Barreto et.al (2013) refieren que es necesario para el ser humano expresar abiertamente su complejidad, dinamismo, autenticidad y espiritualidad como parte de sus recursos y necesidades espirituales. Es la aceptación abierta de la propia espiritualidad que ejerce influencia directa en la inteligencia emocional.

No obstante, Daghan (2017) sustenta que es imprescindible para las enfermeras cuidar de sí mismas a través de la autoconciencia, la autorreflexión y el desarrollo de un sentido de satisfacción para que así brinden mejor atención de enfermería espiritual; sin embargo, Bennett y Thompson, (2015) mencionan que la mejor forma de enseñar a brindar un cuidado espiritualidad a los alumnos es promover la propia espiritualidad del estudiante.

En el presente estudio, observamos que los estudiantes de enfermería poseen un nivel alto de espiritualidad, y no hubo diferencia con esta dimensión, por lo que los resultados hallados concuerdan con la teoría al demostrar que existe correlación las necesidades espirituales y el cuidado humanizado.

Declaración de financiamiento y de conflictos de interés:

El estudio fue financiado por los autores, quienes declaran no tener conflictos de interés 


\section{Correspondencia}

Glendy Larico Calla

Correo electrónico:

glendylarico@gmail.com

\section{Dennis Mamani Quispe \\ Correo electrónico: \\ dennisvmq@gmail.com}

\section{REFERENCIAS BIBLIOGRÁFICAS}

Albornoz, V., \& Chiquez, J. (2018). Condiciones laborales y habilidad del cuidado humanizado en el enfermero/a del Hospital Nacional Daniel Alcides Carrión, Lima 2018. Universidad Privada Norbert Wiener

Alva, L. y Castillo, L. (2018). Espiritualidad y estilo de vida en estudiantes de Medicina Humana de la Universidad Peruana Unión, Lima 2017. (Tesis de licenciatura, Universidad Peruana Unión).

Anchorena, S. (2014). Diferencia de los niveles de inteligencia emocional-social entre los estudiantes católicos practicantes y no practicantes de un colegio privado. Revista de Investigación en Psicología, 17 (2), 159-170. Recuperado de http:// revistasinvestigacion.unmsm.edu.pe/index.php/ psico/article/view/11264/10106 Annual Review of Psychology, 59, 507-536

Arikhman, N., Machmud, R., Hasan,A ., y Bachtiar. H. (2016). Dimension Model Development of Health Center Staff Soft Skills in Mediating Determinant Factors With Performance. International Journal of Scientific Research And Education, 4(11), 6032-6039. doi: http://dx.doi.org/10.18535/ijsre/ v4i11.06

Babamohamadi, H., Ahmadpanah, S. y Ghorbani, R. (2018). Attitudes toward spirituality and spiritual care among Iranian nurses and nursing students: a crosssectional study. Journal of Religion and Health, 57(4), 1304-1314. https://doi. org/10.1007/s10943-017-0485-y

Barreto, P., Fombuena, M., Diego, R., Galiana, L., y Oliver, A. (2013). Medicina Paliativa Bienestar emocional y espiritualidad al final de la vida. Medicina Paliativa, 22(1), 25- 32. doi: 10.1016/j. medipa.2013.02.002

Bennett, V., y Thompson, M. L. (2015). Teaching spirituality to student nurses. Journal of Nursing Education and Practice, 5(2), 22-36. https://doi. org/10.5430/jnep.v5n2p26

Calsin, M. (2017). Percepción de las usuarias sobre el cuidado humanizado que brinda el profesional de
Enfermería en el servicio de Gineco - Obstetricia del Hospital Regional Manuel Núñez Butrón, Puno - 2016. (Tesis de licenciatura, Universidad Nacional del Altiplano). Recuperado de http:// repositorio.unap.edu.pe/handle/UNAP/3873

Casimiro, Z., y Palma, D. (2016). Calidad de cuidados humanizados que brinda el profesional de Enfermería en los servicios de hospitalización de un Hospital Público de Huánuco- 2016. (Tesis de licenciatura, Universidad de Huanuco). Recuperado de https://core.ac.uk/ reader/84496926

Daghan, S. (2017). Nursing students' perceptions of spirituality and spiritual care; an example of Turkey. Journal of Religion and Health, 57(1), 420 -430. https://doi.org/10.1007/s10943-017-0416-y

Evangelista, B., Lopes, L., Costa, G., Abrão, F., Batista, de S. y Oliveira, C. (2016). Spirituality in patient care under palliative care: A study with nurses. Escola Anna Nery - Revista de Enfermagem, 20(1), 176-182. https://doi. org/10.5935/14148145.20160023

Landman, A. (2016). Habilidades para el cuidado humanizado de estudiantes de enfermería. Enfermería: Cuidados Humanizados, 5(1), 29-34. Disponible en https://revistas.ucu.edu. uy/index.php/enfermeriacuidadoshumanizados/ article/view/1190/1154 Cabana, I., y Delgado, J. (2016). Estresores laborales y percepción de autoeficacia del cuidado humanizado en enfermeros, servicio de emergencia, hospital III Yanahuara EsSalud, 2016. (Tesis de licenciatura, Universidad Nacional de San Agustín de Arequipa). Recuperado de http://repositorio. unsa.edu.pe/handle/UNSA/4376

Lujan, D., y Moisés, B. (2014). Nivel de bienestar espiritual en profesionales de Enfermería asistenciales del Hospital Regional de Ayacucho, 2013. (Tesis de licenciatura, Universidad Alas Peruanas). Recuperado de http://repositorio.uap. edu.pe/handle/uap/1338

Melgar, M., y Estuco, J. (2014). Relación de espiritualidad y escala de valores de la enfermería con la percepción del paciente en el Servicio de Emergencia del Hospital Honorio Delgado. Arequipa - 2014. (Tesis de licenciatura, Universidad Nacional de San Agustín). Recuperado de http://repositorio.unsa.edu. pe/bitstream/handle/UNSA/3185/ENSmealmj. pdf? sequence $=1$ \&isAllowed $=y$

Miguel, J., y Vilchez, E. (2018). Inteligencia emocional y espiritualidad en estudiantes universitarios ayacuchanos. (Tesis de licenciatura, Universidad Peruana de Ciencias Aplicadas). Recuperado de http://hdl.handle.net/10757/624060 
Monje V., P., Miranda C., Oyarzün G., Seguel P., Flores G., Monje V., ... Flores G. (2018). Percepción de cuidado humanizado de Enfermería desde la perspectiva de usuarios hospitalizados. Ciencia y Enfermería, 24, 1-10. https://doi.org/10.4067/ S0717-95532018000100205

Paccotaipe, S. (2018). Percepción Sobre Cuidado Humanizado De Enfermería En Pacientes Atendidos En Hospitales Nacionales De Lima Norte. Revista Peruana Salud Pública Comunitaria, 1(2), 78-79. https://doi.org/10.1016/ S2007-5057(14)72746-2

Paredes, Á. (2017). Ética cristiana en la enfermería. Lima - Perú: Fondo Editorial Universidad Peruana Unión.

Parsian, N., y Dunning, T. (2009). Developing and Validating a Questionnaire to Measure Spirituality: A Psychometric Process. Global Journal of Health Science, 1(1),2-11. Recuperado de http:// www.ccsenet.org/journal/index.php/gjhs/article/ view/1104

Poblete, M., Valenzuela, S. y Merino, J. (2012). Validación de dos escalas utilizadas en la medición del cuidado humano transpersonal basadas en la Teoría de Jean Watson. Aquichan, 12(1), 8-21. Recuperado de https://aquichan. unisabana.edu.co/index.php/aquichan/article/ view/1647/2688

Quintero, M. C. (2001). Enfermería en el mundo cristiano. Aquichan, 1(1), 4. Disponible en http://www.scielo.org.co/scielo.php?script=sci arttext\&pid=S1657-59972001000100012
Rodríguez, A. y Honores, A. (2016). Cuidado humanizado que brinda la enfermera en emergencia del Hospital Víctor Lazarte Echegaray, Trujillo, 2016. (Tesis de licenciatura, Universidad Privada Antenor Orrego). Recuperado de http:// repositorio.upao.edu.pe/handle/upaorep/2746

Ross, L., McSherry, W., Giske, T., van Leeuwen, R., Schep-Akkerman, A., Koslander, T., ... Jarvis, P. (2018). Nursing and midwifery students' perceptions of spirituality, spiritual care, and spiritual care competency: A prospective, longitudinal, correlational European study. Nurse Education Today, 67(May), 64-71. https://doi. org/10.1016/j.nedt.2018.05.002

Salgado, A. (2014). Revisión de estudios empíricos sobre el impacto de la religión, religiosidad y espiritualidad como factores protectores. Propósitos y Representaciones, 2(1), 121-159. http://dx.doi.org/10.20511/pyr2014.v2n1.55

Silva, J. (2015). A espiritualidade no cuidado em saúde: CONCEPÇÕES de estudantes de Medicina e de Emfermagem. (Tesis de licenciatura, Universidade Federal da Paraíba). Recuperado de https://repositorio.ufpb.br/jspui/ handle/tede/7874

White, E. (2012). El ministerio de curación. Lima: Servicio Educacional Hogar y Salud.

Recibido: 18/08/2020

Aceptado: 26/10/2020 